\title{
Successful Closed Reduction of a Dislocated Constrained Total Hip Arthroplasty
}

\author{
Motoki Sonohata ${ }^{\dagger}$, Warit Waewsawangwong ${ }^{\sharp}$, Stuart B. Goodman ${ }^{\beta}$
}

This case report and literature review examines whether closed reduction is a viable option to manage hip dislocation when the patient has a constrained liner.

\section{Introduction}

Dislocation after total hip arthroplasty (THA) ranges from less than $1 \%$ to $6 \%$ of primary cases [1] and from $15 \%$ to $30 \%$ of revision cases [2]. Unfortunately, the success rate of non-operative treatment of dislocation after THA can be unreliable, and a third of such patients have recurrences [1].

Surgical procedures used to treat instability and dislocation include:

- Tightening the abductor musculature

- Removing sources of impingement

- Repositioning malaligned components

- Using acetabular liners with elevated rims

Such treatments fail in $30 \%$ to $50 \%$ of patients, however [3].

The use of constrained liners, which relies on a locking mechanism to capture the femoral head, has been developed to help manage this problem [4]. Despite such efforts, patients continue to be at high risk for instability, and 3\% to $18 \%$ experience recurrent dislocation even after constrained components have been implanted $[5,6]$. Generally, open surgical reduction is thought to be the only available treatment for such cases. However, this compromises the integrity of the joint and exposes the patient to the additional risk of surgery [7]. Several authors have reported closed reduction for dislocation of a constrained liner [7-13].

Constrained acetabular liners are currently avail- able in various designs, with differences in the locking mechanisms. To our knowledge, ours is the first reported case of a successful closed reduction of a dislocated constrained THA using a Trilogy Acetabular System Constrained liner (Zimmer, Inc, Warsaw, Indiana, USA).

The study protocol adhered to the ethics guidelines of the 1975 Declaration of Helsinki, and the study was approved by the Institutional Review Board of Stanford University.

\section{Case Report and Management}

A 79-year-old female underwent primary THA for nonunion of a right subtrochanteric fracture of the femur with degenerative arthritis of the right hip joint using an uncemented Trilogy acetabular system and fully porous-coated stem (Zimmer, Warsaw, Indiana, USA). Five years later, at age 84 , the hip was revised to a Trilogy constrained liner with a $10^{\circ}$ oblique face and 32-mm head (Zimmer, Inc, Warsaw, Indiana, USA) (Figure 1) for instability and recurrent dislocation (Figure 2).

However, 5 years after the revision surgery, at age 88 , she bent over, hyperflexed the hip, and complained of right hip pain. A radiograph in the emer-

$\dagger$ Department of Orthopaedic Surgery, Stanford School of Medicine, Stanford University, Stanford, California, USA, and the Department of Orthopedic Surgery, Faculty of Medicine, Saga University, Saga, Japan

$¥$ Department of Orthopaedic Surgery, Stanford School of Medicine, Stanford University, Stanford, California, USA, and the Department of Orthopedic Surgery, Somdej Prapinkao Hospital, Bangkok, Thailand

B Department of Orthopaedic Surgery, Stanford School of Medicine, Stanford University, Stanford, California, USA 


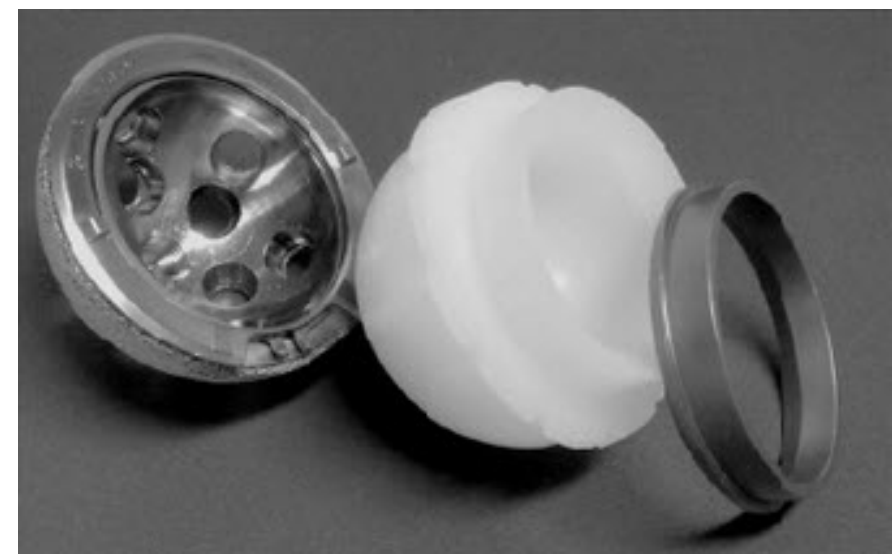

Figure 1. Trilogy constrained liner with a 10-degree oblique face (Zimmer, Inc, Warsaw, Indiana, USA).
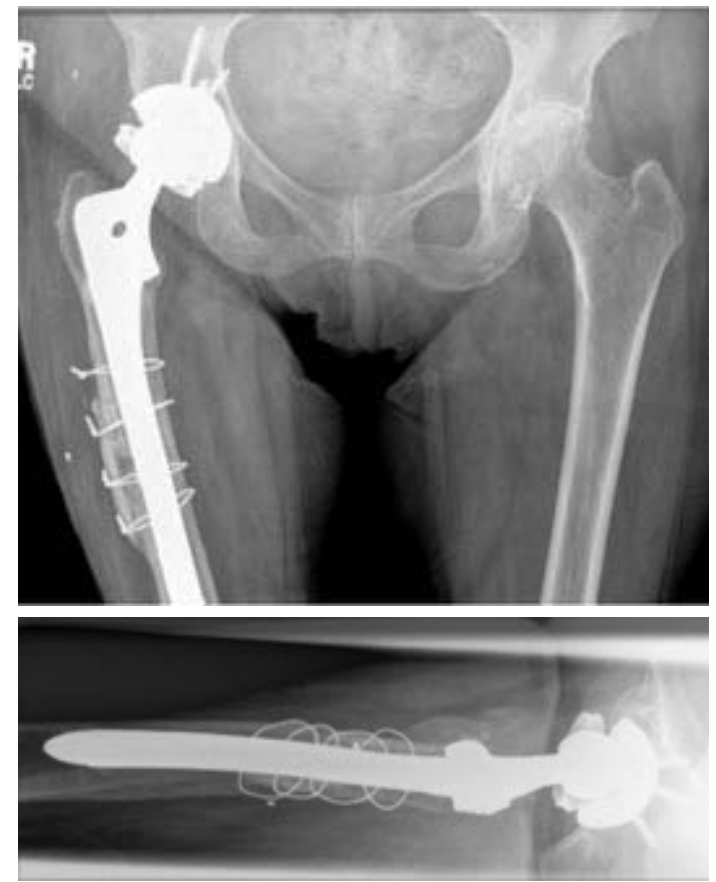

Figure 2. Radiographs before dislocation. (A) Anteroposterior view, (B) Lateral view.

gency department in our hospital demonstrated dislocation of the constrained THA. The head appeared to have dislocated from the liner, and the locking ring disengaged but was not broken (Figure 3).

A closed reduction was performed in the emergency department with sedation. The head was relocated and the locking ring was repositioned to where it was previously located (Figure 4). The patient's hip was placed in an abduction brace with full weight bearing. Further radiographs demonstrated no further change. The patient could ambulate with minimal aid.

The patient died 10 months later of unrelated causes.

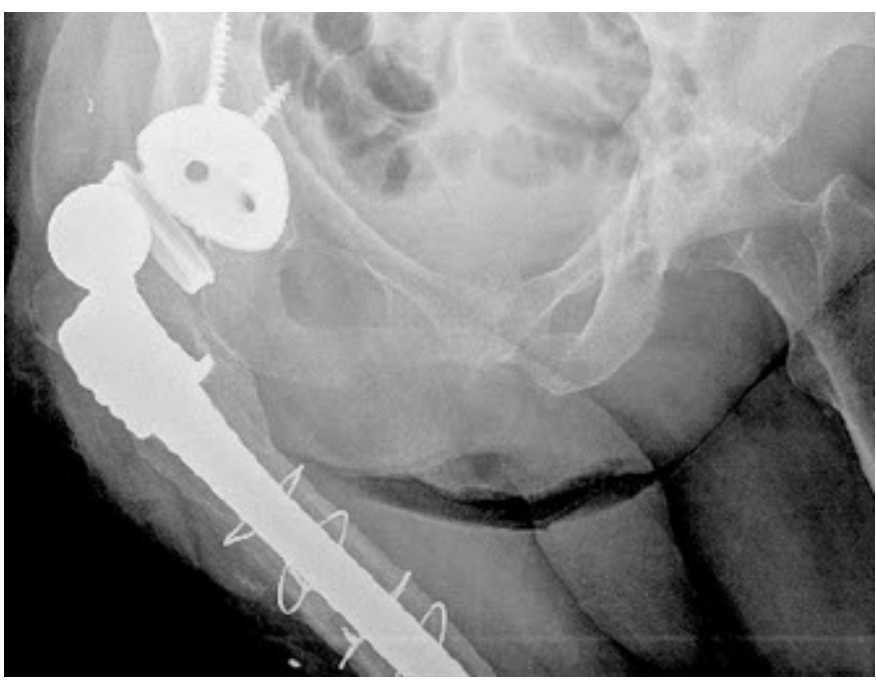

Figure 3. Anteroposterior radiograph of dislocation of the right hip.

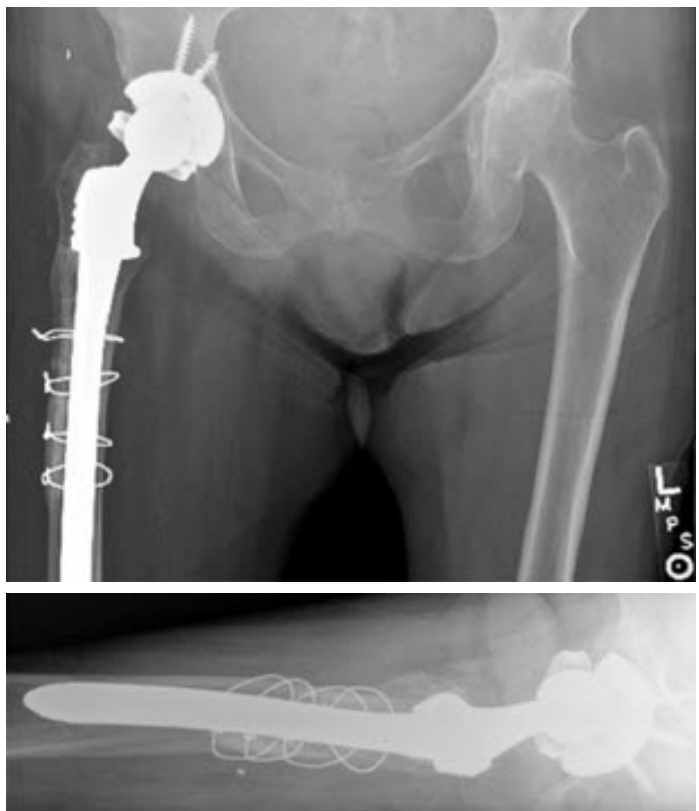

Figure 4. Radiographs after closed reduction. (A) Anteroposterior view, (B) Lateral view.

\section{Discussion}

Dislocation after THA using a constrained polyethylene liner presents a substantial treatment challenge. Closed reduction provides a conservative treatment option that avoids the risk of open surgical reduction. This point is especially relevant considering the surgical complications that exist in older patients with recurrent instability and a complex revision history [9].

Several authors have described successful closed reduction of a dislocated constrained liner [7-13]. Generally, it is thought that plastic deformation must occur in the polyethylene during the initial disloca- 
tion and during the closed reduction process. The torque required for subsequent dislocations for a second and third time decreased by $24 \%$ and $8 \%$ in the Poly-Dial constrained liner (DePuy, Warsaw, Indiana, USA), respectively [9].

However, not all patients undergoing closed reduction of constrained liners require revision surgery or experience recurrent dislocations. Harman et al [9] reported that six hips remained stable 7 to 72 months after the last reduction. They noted that two of six hips underwent repeated successful closed reduction within 6 months of their initial dislocation and reduction and remained stable for at least 3 years.

In contrast, McPherson et al [7] reported that three hips with constrained liners required further operation after closed reduction. Two patients underwent revision surgery after 1 and 3 months their initial dislocation and reduction, and one patient was converted to a Girdlestone resection arthroplasty after 6 months.

Our current case underwent closed reduction once, and did not require any further surgery.

Closed reduction techniques for dislocated constrained liner were reported in seven papers [7-13]. Adequate anesthesia and usage of fluoroscopy were common described in these papers. The procedure of closed reduction for dislocated liner was formed in two steps.

- First, traction was applied and the femoral head was placed in a "perched" position on the acetabular cup. The hip was positioned in 10-40 degrees abduction during traction [7,9-12]. Additional hip flexion was described in two papers, in full extension in one [9]; however, Flint et al adopted in-line traction for the limb [13].

- Second, the femoral head should be passed through the constrained liner with axial compression after confirmation that the femoral head was perched just lateral to the rim. In this phase, hip flexion was increased to 30-90 degrees from first step $[7,9,11]$.

Direct medial pressure was also placed to the greater trochanter [7,9,10,13]. Gaines et al [12] reported an anomalous closed reduction. They initially failed at closed reduction; however, the patient's femoral head subsequently reduced naturally the next day.

The most commonly used femoral heads for constrained liners are $28 \mathrm{~mm}$ and $32 \mathrm{~mm}$ in diameter. Harman et al [9] reported no successful closed reductions of a 32-mm femoral head in their eight patients.
They described that the force to relocate the 28 $\mathrm{mm}$ heads into constrained liners in vitro was 1380 $\mathrm{N}$ (310 Ib), whereas 32-mm heads required greater force. Six 28-mm and one 32-mm head were relocated into constrained liners [9-13]; however, the details of implants and clinical results at follow-up concerning of the case of 32-mm head were unclear [10].

In the current case, the patient' femoral head was 32- $\mathrm{mm}$ head with Trilogy constrained liner (Zimmer, Warsaw, Indiana, USA).

Four constrained liners are currently in common use:

- Omnifit liner (Stryker, Mahwah, New Jersey, USA)

- S-ROM liner/Poly-Dial (Depuy, Warsaw, Indiana, USA)

- RingLoc constrained acetabular liner (Biomet, Warsaw, Indiana, USA)

- Trilogy constrained liner (Zimmer, Warsaw, Indiana, USA)

Given substantial differences among constrained components from different implant manufactures, several reported closed reduction techniques may not be applicable to specific constrained acetabular components.

Our case is the first report of successful closed reduction of a dislocated constrained total hip arthroplasty using the Trilogy constrained liner and a $32-\mathrm{mm}$ head. In the aging population with complex medical issues, closed reduction may obviate the need for a more invasive open procedure, or at least restore patient function until revision THA can be electivity planned. An attempt at closed reduction may be indicated unless the acetabular component has failed at the liner-shell interface, shell-bone interface, or the locking ring has fractured.

The limitation of this case report was that the follow-up period was only 10 months. Therefore, it is unclear whether recurrences of dislocation might happen with longer-term follow up.

\section{Source}

Sonohata M, Waewsawangwong W, Goodman, SB. Successful Closed Reduction of a Dislocated Constrained Total Hip Arthroplasty: A Case Report and Literature Review. The Open Orthopaedics Journal, 2012, 6, 211-214. doi: 10.2174/1874325001206010211. http://benthamscience.com/open/openaccess.php?toorthj/articles/ V006/211TOORTHJ.htm

(C) Sonohata et al; Licensee Bentham Open. This 
is an open access article licensed under the terms of the Creative Commons Attribution Non-Commercial License (http://creativecommons.org/licenses/bync/3.0/) which permits unrestricted, non-commercial use, distribution and reproduction in any medium, provided the work is properly cited.

\section{References}

1. Soong M, Rubash HE, Macaulay W. Dislocation after total hip arthroplasty. J Am Acad Orthop Surg 2004; 12(5): 314-21.

2. Su EP, Pellicci PM. The role of constrained liners in total hip arthroplasty. Clin Orthop 2004; 420: 122-9.

3. Morrey BF. Difficult complications after hip joint replacement. Dislocation. Clin Orthop 1997; 344: 179-87.

4. Shrader MW, Parvizi J, Lewallen DG. The use of a constrainedacetabular component to treat instability after total hip arthroplasty. J Bone Joint Surg Am 2003; 85(11): 2179-83.

5. Berend KR, Lombardi AV Jr, Mallory TH, Adams JB, Russell JH,Groseth KL. The long-term outcome of 755 consecutive constrained acetabular components in total hip arthroplasty examining the successes and failures. J Arthroplasty 2005; 20(7 Suppl. 3): 93-102.
6. Khan RJ, Fick D, Alakeson R, et al. A constrained acetabular component for recurrent dislocation. J Bone Joint Surg Br 2006; 88(7): 870-6.

7. McPherson EJ, Costigan WM, Gerhardt MB, Norris LR. Closed reduction of dislocated total hip with S-ROM constrained acetabular component. J Arthroplasty 1999; 14(7): 882-5.

8. Miller CW, Zura RD. Closed reduction of a dislocation of aconstrained acetabular component. J Arthroplasty 2001; 16(4): 504-5.

9. Harman MK, Hodge WA, Banks SA. Closed reduction of constrained total hip arthroplasty. Clin Orthop 2003; 414: 121-8.

10. Birdwell S, Wilke E. Closed reduction of constrained total hip arthroplasty in the ED. J Emerg Med 2011; 40(2): 162-6.

11. Robertson WJ, Mattern CJ, Hur J, Su EP, Pellicci PM. Failure mechanisms and closed reduction of a constrained tripolar acetabular liner. $\mathbf{J}$ Arthroplast 2009; 24(2): 322.e5-11.

12. Gaines RJ, Hardenbrook M. Closed reduction of a dislocated total hip arthroplasty with a constrained acetabular component. Am J Orthop (Belle Mead NJ) 2009; 38(10): 523-5.

13. Flint JH, Phisitkul P, Callaghan JJ. Closed reduction of a dislocated constrained total hip arthroplasty using a novel technique with a peg board. Orthopedics 2010; 10: 201-3.

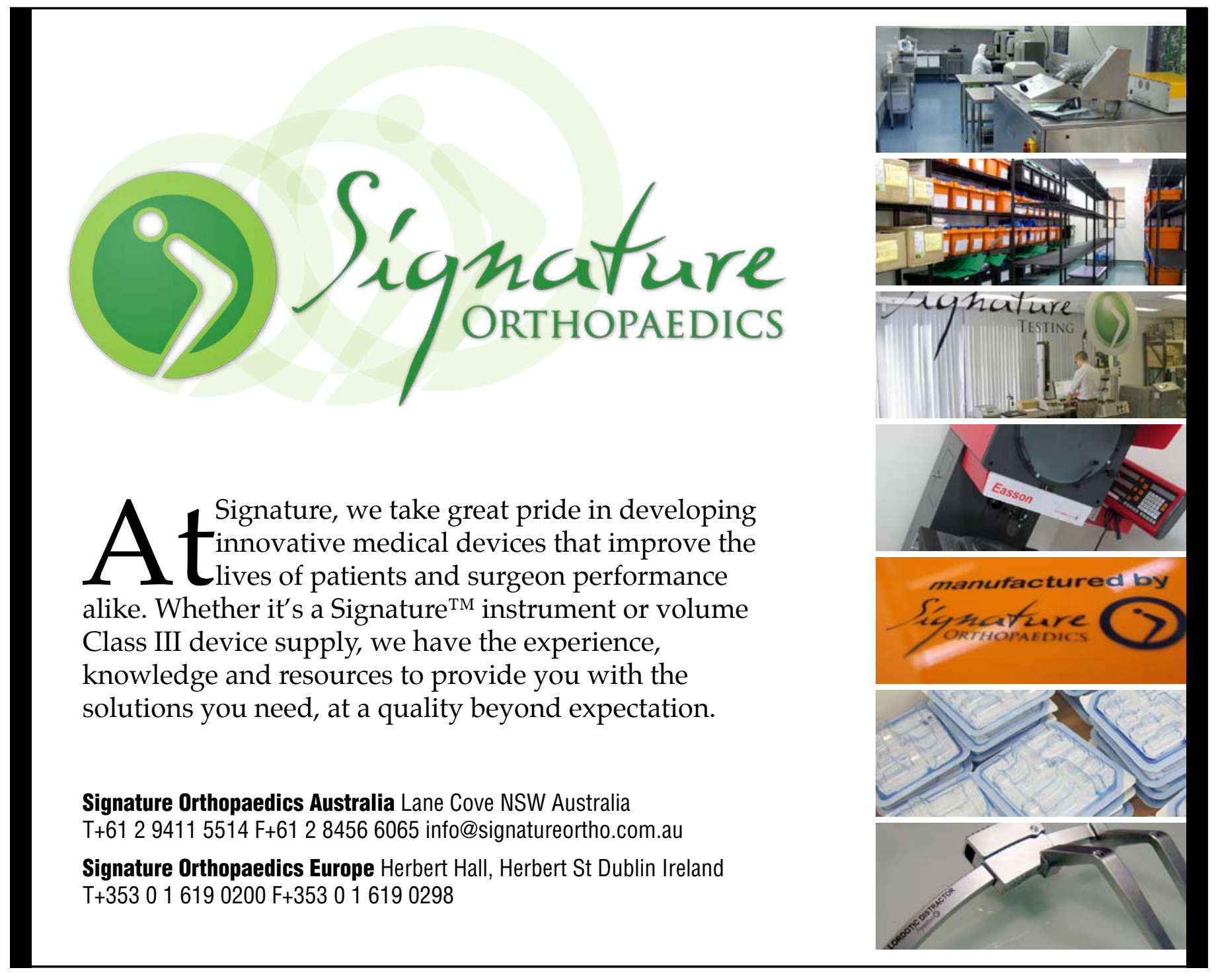

